\title{
Surgical-Orthodontic Management of Mandibular Hypoplasia with Severely Short-Rooted Dentition Secondary to Anti-Leukemic Treatment: A Case Report
}

\author{
Supakit Peanchitlertkajorn*, Kallaya Wangpichit and Ladda Winalukwong
}

Baylor College of Dentistry, University of Texas Southwestern Medical Center, Dallas, TX, USA

\begin{abstract}
Major advances in anti-leukemic treatment have increased a significant number of pediatric cancer survivors over the past few decades. Such treatment in children is also reported to cause abnormal dentofacial development including a significant mandibular hypoplasia, dental anomalies, and severely short-rooted dentition. These problems, despite a becoming both functional and psychosocial problems, are often neglected as many clinician's lack experience in managing them. They are exacerbated by the fact that orthodontic treatments can potentially cause further root shortening. Therefore, a special consideration to minimize a risk of root resorption must be given when an orthodontic treatment plan is formulated for these patients. We report a unique treatment approach employed on a 14-year-old male patient with severe dentofacial abnormalities secondary to anti-leukemic treatment. Treatment goals were to correct dental malocclusion, optimize facial balance, and also prevent further root shortening. A treatment plan combining orthodontics, orthognathic surgery, and use of temporary anchorage devices, was developed so that light orthodontic forces could be used throughout the course of treatment. Consequently, the lighter force reduced the risk of potential root resorption. The final outcome demonstrates that the surgical-orthodontic approach optimizes facial and occlusal changes while minimizing further root resorption.
\end{abstract}

\section{Introduction}

Leukemia is the most common form of pediatric malignancy and constitutes approximately $26 \%$ of pediatric cancers [1]. Acute lymphoblastic leukemia (ALL) accounts for $75 \%$ of childhood leukemia [2]. ALL is a malignant disorder of lymphoid progenitor cells. The disease affects both children and adults. It has a peak prevalence between 2 and 5 years of age [3]. The disease can originate in lymphoid cells of different lineages; B-cell ( $85 \%$ of pediatric ALL), T-cell (10\% to $15 \%$ of pediatric ALL), and mixed lineage [4]. Personalized chemo-radiation therapy and improved supportive care have proven effective in treating leukemia. Almost $90 \%$ of children diagnosed with leukemia will survive for more than 5 years if proper treatment is administered [5]. These therapeutic improvements have resulted in an estimated 380,000 pediatric cancer survivors in the US [1]. Chemo-radiation therapy and bone marrow transplant in pediatric patients, are reported to cause abnormal dentofacial developments. Such developments include enamel hypoplasia, microdontia, hypodontia, tooth agenesis, anomalous root development, significant mandibular hypoplasia, increased periodontal inflammation, and reduced salivary flow [6-13]. These treatment-related consequences are common and may not manifest until years later. They are often neglected sources of morbidity and reduce health-related quality of life. A significant number of long-term leukemia survivors with abnormal dentofacial development, secondary to cancer treatments, require orthodontic treatment. Orthodontic treatment for these patients can pose significant challenges including pre-existing short roots and other severe dentofacial anomalies. Special consideration to minimize further root shortening must be given when formulating an orthodontic treatment plan for these patients as orthodontic treatment is reported to potentially cause root resorption [14].

As previously discussed, the detrimental side effects of anti-cancer therapy on dentofacial development have been well documented. However, there are very few articles in the literature that directly address orthodontic treatment in ALL survivors. These articles are mainly case reports and case series $[9,15,16]$. One common discussion among these reports was a concern over pre-existing anomalous root forms and an increased risk for further shortening. A few recommendations were re- ported to minimize the potential root shortening. They included using lighter orthodontic forces, terminating the treatment earlier than normal, choosing the simplest method for the treatment needs, and leaving lower dentition and mandible untreated. However, the treatment results were deemed unsatisfactory due to compromised outcomes [9]. In a recent questionnaire concerning experience and expertise in orthodontic treatment of pediatric cancer survivors among orthodontists, $85 \%$ of respondents replied that their orthodontic education did not sufficiently prepare them for such treatment [17].

The purpose of this report is to present a unique treatment approach combining orthodontic and surgical treatment for a patient with mandibular hypoplasia with severe pre-existing short roots secondary to leukemia treatment. The treatment goals are not only to treat dental malocclusion and optimize facial balance, but also to prevent further root shortening.

\section{Etiology and Diagnosis}

A 14-year-old male patient with a history of leukemia was referred to our clinic for an orthodontic evaluation. He was diagnosed with Acute Lymphoblastic Leukemia at the age of 2 years old. He had been treated with chemo-radiation therapy and bone marrow transplant from the time of diagnosis until 9 years of age. He had been in remis-

*Corresponding author: Supakit Peanchitlertkajorn D.D.S., M.D.S, Former Clinical Assistant Professor at Baylor College of Dentistry, University of Texas Southwestern Medical Center, Dallas, TX, USA, Tel: 5105817851; E-mail: supakit@att.net

Received: May 22, 2016; Accepted: June 12, 2017; Published: June 16, 2017

Citation: Peanchitlertkajorn S, Wangpichit K, Winalukwong L (2017) SurgicalOrthodontic Management of Mandibular Hypoplasia with Severely Short-Rooted Dentition Secondary to Anti-Leukemic Treatment: A Case Report. Oral health case Rep 3: 136. doi:10.4172/2471-8726.1000136

Copyright: $\odot 2017$ Peanchitlertkajorn S. This is an open-access article distributed under the terms of the Creative Commons Attribution License, which permits unrestricted use, distribution, and reproduction in any medium, provided the original author and source are credited. 
Citation: Peanchitlertkajorn S, Wangpichit K, Winalukwong L (2017) Surgical-Orthodontic Management of Mandibular Hypoplasia with Severely ShortRooted Dentition Secondary to Anti-Leukemic Treatment: A Case Report. Oral health case Rep 3: 136. doi:10.4172/2471-8726.1000136

Page 2 of 5

sion for 5 years at the time of referral. His chief complaint was crowded teeth. He had a retrognathic profile with normal nasolabial angle and deep mentolabial sulcus. The lower facial height was short. Dentally, he presented with severe anterior crowding of upper and lower anterior teeth. The upper right primary canine was still present, but both upper and lower right permanent canines were clinically missing. The upper left permanent canine was labially displaced as a result of the over-retained upper left primary canine. The lower left and right permanent central incisors were displaced lingually due to the over-retained lower left and right primary central incisors. The lower left and right permanent lateral incisors were clinically missing. The overjet was recorded at $5 \mathrm{~mm}$ with $60 \%$ overbite. The molar relationships were Angle's Classification II bilaterally (Figure 1).

Radiographic examination revealed an impacted upper right canine, an unerupted lower right canine, congenitally missing lower left and right lateral incisors, microdontia of all third molars, and severely short-rooted dentition as a result of anti-cancer therapy (Figure 2). Cephalometric analysis revealed that the patient had a Class II skeletal pattern with an ANB angle of $4^{\circ}$. The SNA was measured at $80^{\circ}$. The mandible was hypoplastic with SNB angle of $76^{\circ}$. The mandibular plane angle was within normal limits $\left(\mathrm{SN} / \mathrm{MP}=23^{\circ}\right)$. The lower anterior facial height was short (ANS-Me/N-Me $\times 100=45 \%$ ). The maxillary and mandibular incisors were slightly proclined $\left(\underline{1}-\mathrm{PP}=120\right.$ degrees, $\left.\mathrm{IMPA}=97^{\circ}\right)$.
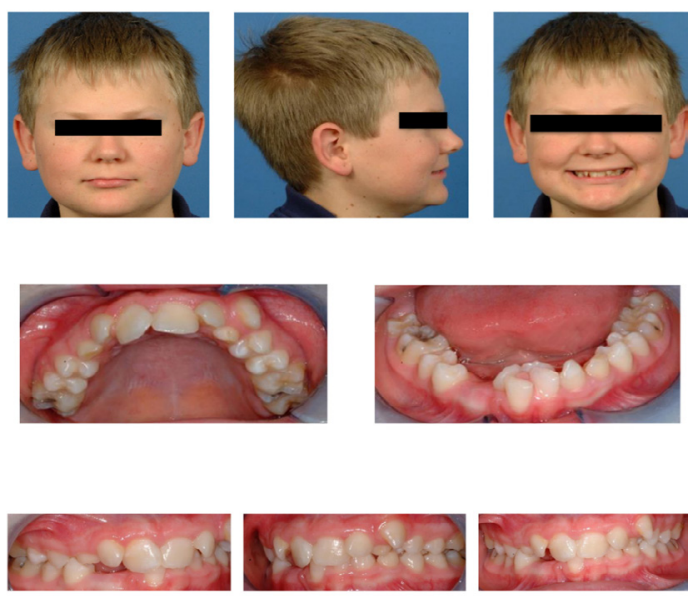

Figure 1: Pre-treatment extraoral and introral pictures.
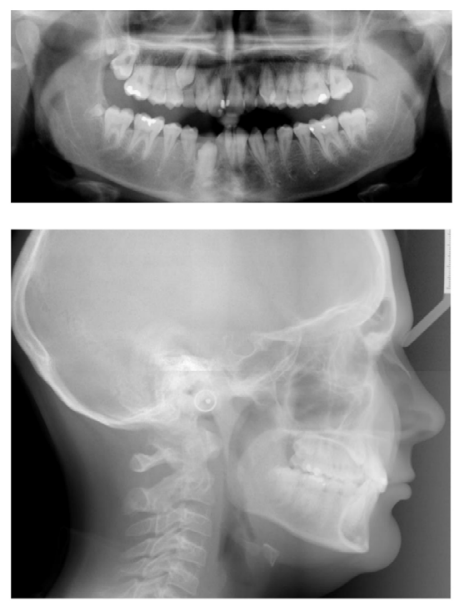

Figure 2: Pre-treatment panoramic and cephalometric radiographs.
These clinical and radiographic findings are negative treatment-related consequences of his previous ALL therapy.

\section{Treatment Objectives and Alternative Treatment Plans}

The treatment objectives were as follows:

- Align all teeth

- Guide the eruption of the impacted canines

- Establish optimal overjet and overbite

- Improve jaw relationship and facial profile

- Minimize root resorption

- Minimize post-orthodontic restoration

Two alternative treatment plans were presented to the patient and his parents. The first treatment option was a combination of orthodontic treatment and orthognathic surgery along with the use temporary anchorage devices (TADs). This plan would employ surgical mandibular advancement to correct the Class II malocclusion and to avoid extensive sagittal orthodontic tooth movement. TADs would be placed during the surgery to serve as anchors so that a surgical splint could be secured directly onto them instead of the teeth. Inter-maxillary elastics could be worn directly from TADs during post-operative care to stabilize the new jaw position. This approach was designed to minimize the amount of orthodontic forces exerted directly on the dentition. Consequently, the lighter orthodontic force used during the treatment would reduce the risk of a potential root resorption.

The second treatment option was to perform orthodontic treatment on the upper teeth only, along with extracting the remaining primary teeth except for the lower left primary canine. The goal for this treatment option was to align the teeth but leave the Class II malocclusion and excessive overjet uncorrected. Pros and cons were discussed at length with the parents and patient. The family chose the first treatment option.

\section{Treatment Progress}

The remaining primary teeth were extracted except the lower left primary canine, which would be preserved until the patient was ready for a dental restoration. Full orthodontic appliances were bonded and banded except for the lower left primary canine. Light orthodontic forces (014 Nitinol archwires) were used for initial leveling and aligning. A few months into treatment, the lower right permanent canine spontaneously erupted. Teeth alignment and space regaining for the upper right canine continued for 17 months. The patient was then referred to an oral surgeon for surgical exposure and bonding of a button and gold chain on the impacted upper right canine. The use of light orthodontic forces was continued to artificially erupt the impacted tooth. The upper right canine erupted after 5 months of orthodontic traction.

After 29 months of treatment, all teeth were fully erupted and well aligned. The patient was then referred to an oral maxillofacial surgeon for an osteotomy to advance the mandible. The orthodontic appliances were removed right before the osteotomy to minimize any undesirable shifting of the teeth (Figures 3 and 4).

The maxillofacial surgeon planned to remove all the third molars at the time of the mandibular osteotomy. Microdontia of the third molars did not increase the risk of post-operative infection. The mandible was advanced $5 \mathrm{~mm}$ at the $\mathrm{B}$ point. The orthognathic surgery was employed on this patient to avoid using orthodontic forces necessary to correct 
Citation: Peanchitlertkajorn S, Wangpichit K, Winalukwong L (2017) Surgical-Orthodontic Management of Mandibular Hypoplasia with Severely ShortRooted Dentition Secondary to Anti-Leukemic Treatment: A Case Report. Oral health case Rep 3: 136. doi:10.4172/2471-8726.1000136

Page 3 of 5

Class II malocclusion (Figures 5 and 6). Temporary anchorage devices (TADs) were placed during the surgery to avoid any forces being directly applied to the teeth during the surgery and post-operatively. Two TADs were placed in each quadrant; one distal to a canine, and the other distal to a first molar. Each was inserted above the root tips. The surgical splint was secured directly to the TADs. Post-surgical elastic bands were also placed on TADs post-surgically. TADs were removed after the jaw position and occlusion became stable. The overall treatment time was 32 months.

\section{Treatment Results}

Facially, the patient achieved a balanced profile with a proportionate lower facial height (Figures 5 and 6). The dentition was well aligned. The left canine and molar relationships were in Class I. The right canine and molar relationships were in Class III but with good interdigitation between upper and lower posterior teeth. The overjet and overbite were within normal range. The final panoramic radiograph showed that all teeth maintained their root lengths (Figure 6). Superimposition of pre-treatment, pre-surgical and post-treatment cephalometric tracings
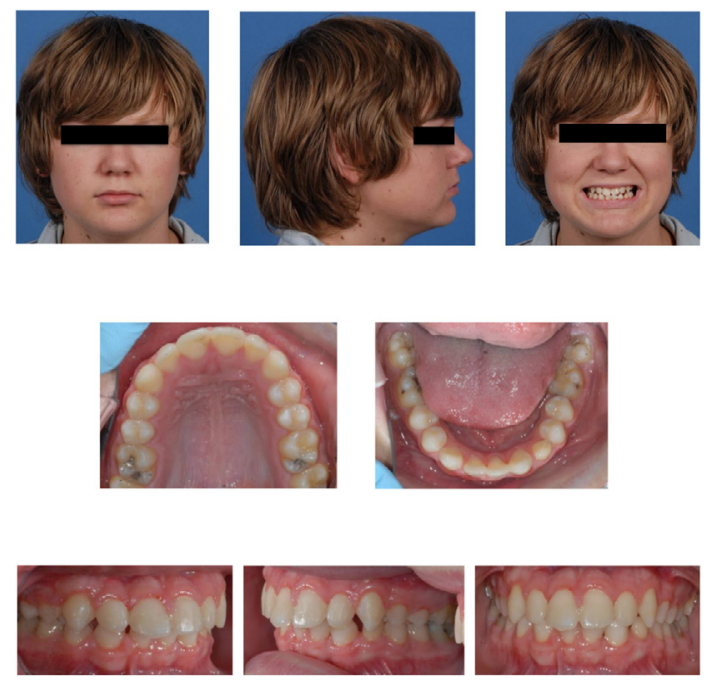

Figure 3: Pre-surgical extraoral and intraoral pictures.
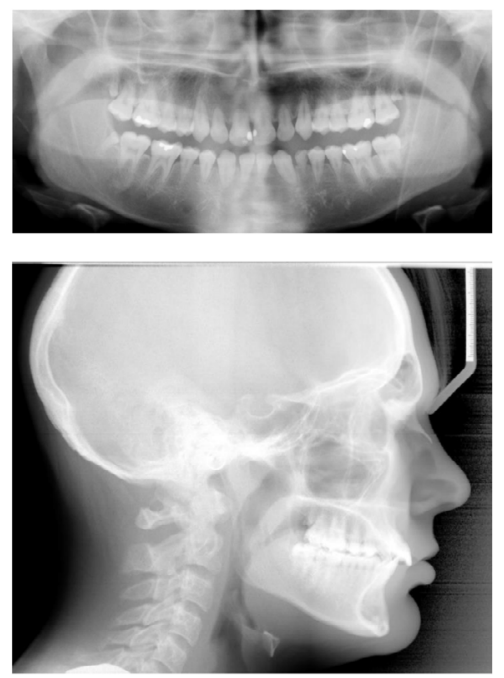

Figure 4: Pre-surgical panoramic and cephalometric radiographs.
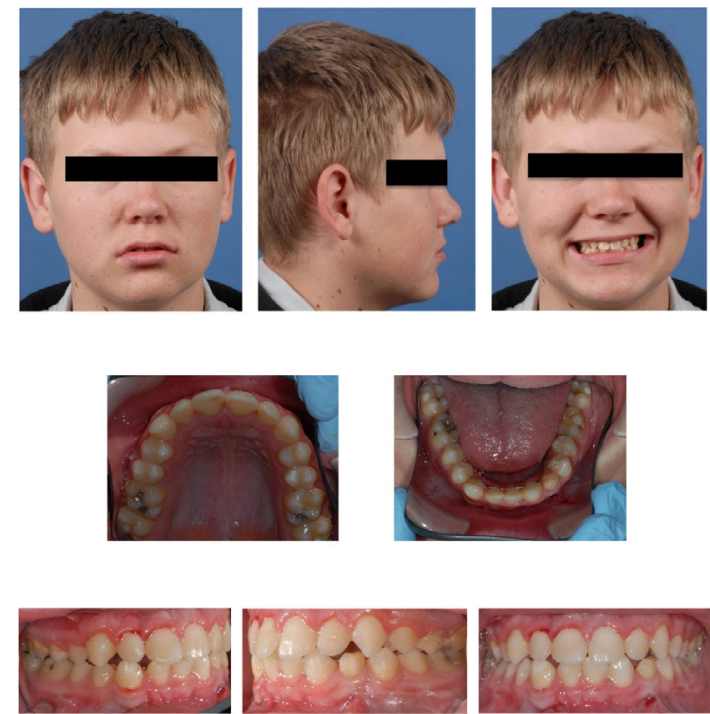

Figure 5: Post-surgical extraoral and intraoral pictures.
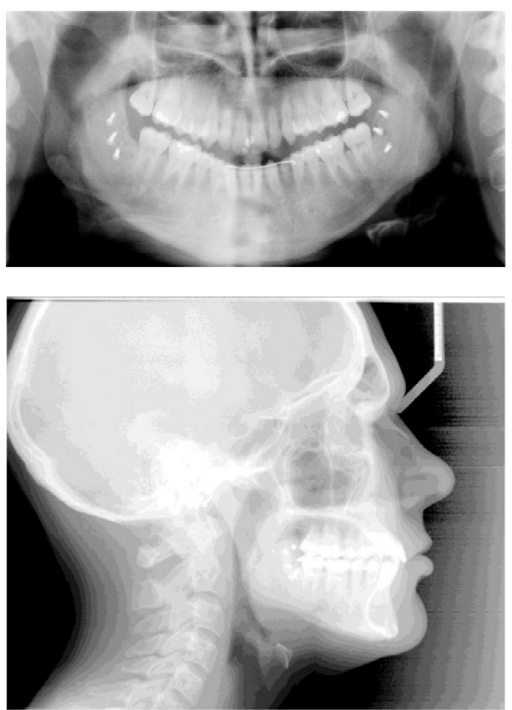

Figure 6: Post-surgical panoramic and cephalometric radiographs.

showed favorable dentofacial changes as a result of the treatment (Figure 7). A dental implant was recommended as a permanent restoration to replace the lower left primary canine upon completion of facial growth [18].

\section{Case Retention}

A maxillary Hawley retainer and a mandibular bonded retainer were used after completing the orthodontic treatment. The lower bonded retainer will be removed and replaced by a Hawley retainer when the patient has a prosthesis replacing the lower primary canine. The patient was counseled to wear retainer for life. A similar retention protocol of 24-32-month retainer use was reported to produce a stable upper anterior alignment and a minor irregularity of the lower incisors [19].

\section{Case Discussion}

As the number of long-term pediatric cancer survivors grows, an increasing number of these patients will seek orthodontic treatment 


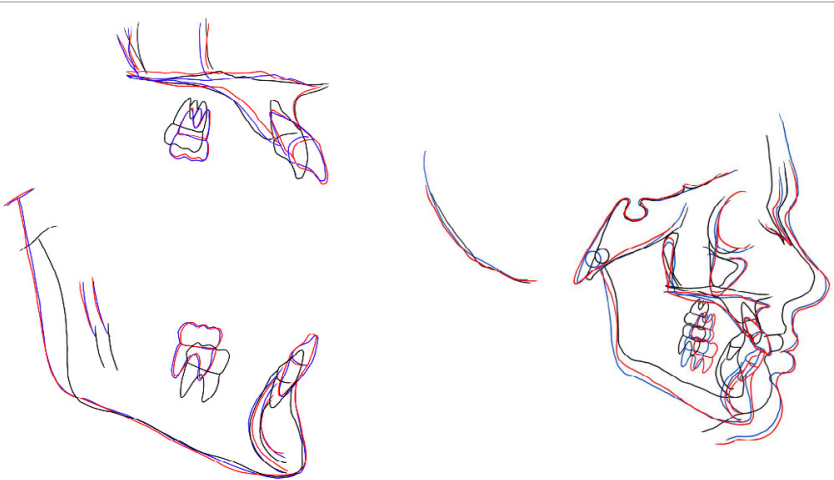

Figure 7: Superimposition of cephalometric tracings (black, pre-treatment; blue, pre-surgical; red, final).

to resolve their dentofacial abnormalities secondary to the anti-cancer treatments they received. Timing of the orthodontic treatment is of essence and so is modifying the treatment to reflect the patient's health condition. Sheller and Williams advised that intervention of an orthodontic treatment should be at least 2 years after the completion of all cancer treatments. In addition, a patient should have at least a 2 -year event-free survival. By this time, the risk for relapse of the disease has significantly diminished [20]. In this case report, our patient was diagnosed at the age of 2 years old. He had been in treatment until the age of 9. The orthodontic treatment commenced more than 5 years after completing his anti-cancer treatments and the patient had been in remission for the same amount of time.

Disturbances in dental and root development secondary to cancer treatments include enamel hypoplasia, microdontia, increased incidence of tooth agenesis, V-shaped roots, arrested root development, and premature apical closure [6-13]. Recent animal studies demonstrated that widely used alkylating drugs in anti-cancer treatment such as Cyclophosphamide and Busulfan produced cytotoxicity and caused detrimental effects on the mitotic activity in the root proliferation zone (Hertwig's epithelial root sheath or odontoblasts). Consequently, the molar roots of these experimental rats developed significantly slower and shorter in length. Additionally, the apical foramen of those roots tended to close faster, forming anomalous root shapes [21-23]. Root resorption is a potential side effect of orthodontic therapy. The degree of root resorption in teeth with blunt or pipette shaped roots was significantly higher than in teeth with a normal root form [14]. Hence this group of patients has an increased risk of developing additional root resorption. Positive correlations exist between increased force levels and increased root resorption [24]. Therefore, the use light orthodontic force during the course of treatment has been emphasized in this group of patients [25].

The clinical and radiographic findings in our patient coincide with findings from studies previously discussed. The oral examination revealed a significant disturbance of dentitional development including anterior dental crowding, over-retained primary teeth, ectopic eruption, and clinically missing teeth. Radiographically, an impacted upper right canine, congenitally missing lower left and right lateral incisors, microdontia of all third molars, and severely short-rooted dentition were found.

Several studies demonstrated the effects of anti-cancer treatment on craniofacial structures. Children undergoing such treatments, including cranial irradiation, exhibited a shortened cranial base, maxillamandibular hypoplasia, and a significant decrease (50\% reduction) in height of the alveolar processes [26,27]. It is also reported that patients younger than 5 years of age at the start of anti-cancer treatment, who received high cranial irradiation, had a significantly increased risk of dentofacial abnormalities and especially of mandibular hypoplasia [27]. The growth repressive action of irradiation in growing children has been explained to be due to growth hormone deficiency [28]. Since radiation therapy has a growth-suppressive effect, the prognosis for treating a skeletal Class II malocclusion with growth modification or orthodontic treatment alone could be poor. The treatment approach for our patient employed orthognathic surgery to correct the mandibular hypoplasia and Class II molar relationship. This approach allowed for a complete correction of the excessive overjet, and created facial profile balance while maintaining the use of light orthodontic force during the treatment. Even though the mandibular osteotomy was performed before the facial growth completely stopped, the risk of any residual growth that could change the final outcome was negligible in this patient due to growth repressive effects of radiation therapy on the facial bones. In an additional effort to avoid heavy forces, the orthodontic appliances were removed right before the orthognathic surgery. TADs were used so that any heavy forces used peri- and post-operatively were directed toward jaw bones instead of teeth.

A dental implant was planned for our patient to replace the lower left primary canine. Dental implants are usually performed when facial growth stops to minimize the possibility for occlusal changes [18]. In a recent systematic review, Chambrone and coworkers reported considerably high percentages of survival rate for dental implants in irradiated patients. Additionally, they also found that radiation therapy was linked to higher implant failure in the maxilla, and hyperbaric oxygen therapy did not reduce the risk of implant failure. However, they concluded that dental implants appear to be a viable treatment option for restoring masticatory functions in irradiated patients [29].

\section{Conclusion}

This article discussed dentofacial abnormalities secondary to antileukemic treatment. An adolescent male, a long-term ALL survivor, presented for orthodontic treatment. A treatment plan, combining orthodontics and mandibular osteotomy, was employed. The plan was formulated to maintain the use of light orthodontic forces to reduce the potential for root resorption. The final outcome demonstrated that the treatment approach optimized facial and occlusal changes while minimizing further root shortening, even though the patient exhibited severe preexisting disturbances in root development.

\section{References}

1. Ward E, DeSantis C, Robbins A, Kohler B, Jemal A (2014) Childhood and adolescent cancer statistics, 2014. CA Cancer J Clin 64: 83-103.

2. Margolin JF, Steuber CP, Poplack DG (2002) Acute lymphoblastic leukemia In: Principles and Practice of Pediatric Oncology (4thedn) Philadelphia, PA Lippincott Williams \& Wilkins; 489-544p

3. Pui CH, Robison LL, Look AT (2008) Acute lymphoblastic leukaemia. Lancet 371: 1030-1043.

4. Bhojwani D, Yang JJ, Pui CH (2015) Biology of childhood acute lymphoblastic leukemia. Pediatr Clin N Am 62: 47-60.

5. Pui CH, Mullighan CG, Evans WE, Relling MV (2012) Pediatric acute lymphoblastic leukemia: Where are we going and how do we get there? Blood 120: $1165-1174$

6. Minicucci E, Lopes L, Crocci A (2003) Dental abnormalities in children after chemotherapy treatment for acute lymphoid leukemia. Leuk Res 27: 45-50.

7. Dahllöf G, Barr M, Bolme P (1998) Disturbances in dental development after total body irradiation in bone marrow transplant recipients. Oral Surg Oral Med Oral Pathol 65: 41-44. 
Citation: Peanchitlertkajorn S, Wangpichit K, Winalukwong L (2017) Surgical-Orthodontic Management of Mandibular Hypoplasia with Severely ShortRooted Dentition Secondary to Anti-Leukemic Treatment: A Case Report. Oral health case Rep 3: 136. doi:10.4172/2471-8726.1000136

Page 5 of 5

8. Näsman M, Forsberg CM, Dahllöf G (1997) Long-term dental development in children after treatment for malignant disease. Eur J Orthod 19: 151-159.

9. Dahllöf G, Jönsson A, Ulmner M, Huggare J (2001) Orthodontic treatment in long-term survivors after pediatric bone marrow transplantation. Am J Orthod Dentofacial Orthop 120: 459-465.

10. Duggal MS (2003) Root surface areas in long-term survivors of childhood cancer. Oral Oncol 39: 178-183.

11. Javed F, Utreja A, Bello-Correa FO (2002) Oral health status in children with acute lymphoblastic leukemia. Crit Rev Oncol Hematol 83: 303-309.

12. Rosenberg SW, Kolodney H, Wong GY, Murphy ML (1987) Altered dental root development in long-term survivors of pediatric acute lymphoblastic leukemia. A review of 17 cases. Cancer 59: 1640-1648.

13. Levander E, Malmgren O (1988) Evaluation of the risk of root resorption during orthodontic treatment: A study of upper incisors. Eur J Orthod 10: 30-38.

14. Runge ME, Edwards DL (2000) Orthodontic treatment for an adolescent with history of acute lymphoblastic leukemia. Pediatr Dent 22: 494-498.

15. Kumar S, Valiathan A, Jayaswal P, Sivakumar (2011) A class II treatment of an adolescent patient with a history of acute lymphocytic leukemia. J Clin Orthod 45: 661-665.

16. Neill C, Miglitorati C, Trojan T, Kaste S, Karydis A (2015) Experience and expertise regarding orthodontic management of childhood and adolescen cancer survivors. Am J Dentofacial Orthop 148: 765-770.

17. Op Heij D, Opdebeeck H, Van Steenberghe D, Quirynen M (2003) Age as compromising factor for implant insertion. Periodontology 33: 172-184.

18. Dyer KC, Vaden JL, Harris EF (2012) Relapse revisited-again. Am J Dentofacial Orthop 142: 221-227.
19. Sheller B, Williams B (1996) Orthodontic management of patients with hematologic malignancies. Am J Orthod Dentofacial Orthop 109: 575-580.

20. Kawakami T, Nakamura Y, Karibe H (2015) Cyclophosphamide inhibits root development of molar teeth in growing mice. Odontology 103: 143-151.

21. Mitomi T, Kawano Y, Kinoshita-Kawano S (2014) Effect of the antineoplastic agent busulfan on rat molar root development. Arch Oral Biol 59: 47-59.

22. Kawakami T, Nakamura Y, Karibe H (2015) Cyclophosphamide-induced morphological changes in dental root development of ICR mice. PLOS ONE 10: e0133256

23. Roscoe MG, Meira JB, Cattaneo PM (2015) Association of orthodontic force system and root resorption: A systematic review. Am J Orthod Dentofacial Orthop 147: 610-626.

24. Levander E, Malmgren O, Eliasson S (1994) Evaluation of root resorption in relation to two orthodontic treatment regimens. A clinical experimental study. Eur J Orthod 16: 223-228.

25. Dahllöf G, Forsberg CM, Ringdén O (1989) Facial growth and morphology in long-term survivors after bone marrow transplantation. Eur $\mathrm{J}$ Orthod 11 : 332-340.

26. Sonis AL, Tarbell N, Valachovic RW, Gelber R, Schwenn M, et al. (1990) Dentofacial development in long-term survivors of acute lymphoblastic leukemia. A comparison of three treatment modalities. Cancer 66: 2645-2652.

27. Sanders JE (2008) Growth and development after hematopoietic cell transplant in children. Bone Marrow Transplant 41: 223-227.

28. Chambrone L, Mandia J Jr, Shibli JA, Romito GA, Abrahao M (2013) Denta implants installed in irradiated jaws: A systematic review. J Dent Res 92: $-130 \mathrm{~S}$ 